Supporting Information

\title{
A Surface Effect on Frictional Properties for Thin Hydrogel Films of Poly(vinyl ether)
}

Nozomi Itagaki,,$^{\dagger}$ Daisuke Kawaguchi, ${ }^{* \dagger, \dagger}$ Yukari Oda, ${ }^{\dagger, *}$ Fumiya Nemoto, ${ }^{\#}$ Norifumi L. Yamada, " Tetsuo Yamaguchi," and Keiji Tanaka*,†,\$,

$†$ Department of Applied Chemistry, Kyushu University, Fukuoka 819-0395, Japan

† Center for Polymer Interface and Molecular Adhesion Science, Kyushu University, Fukuoka 819-0395, Japan

\# Neutron Science Laboratory, High Energy Accelerator Research Organization, Naka, Ibaraki 319-1106, Japan

ฯ Department of Mechanical Engineering, Kyushu University, Fukuoka 819-0395, Japan

$\S$ International Institute for Carbon-Neutral Energy Research (WPI-I2CNER), Kyushu University, Fukuoka 819-0395, Japan 


\section{Details for atomic and lateral force microscopy}

A rectangular-shaped cantilever with a spherical colloidal probe made of a borosilicate glass with a diameter of approximately $5 \mu \mathrm{m}$ (CP-CONT-BSG-A, sQUBE, Bickenbach, Germany) was used for atomic and lateral force microscopy (AFM and LFM). The resonance frequency of the cantilever in water was $401 \mathrm{kHz}$. To minimize the deformation of the sample surface during the observation, the set point amplitude was maintained at $0.92(8 \%$ damping of the free oscillation amplitude).

The dependence of lateral force $\left(F_{\mathrm{L}}\right)$ on normal force $\left(F_{\mathrm{N}}\right)$ was examined by LFM measurements at a constant scanning rate of $80 \mu \mathrm{m} \bullet \mathrm{s}^{-1}$ for a travel distance of $20 \mu \mathrm{m} . \quad F_{\mathrm{L}}$ was calculated from a frictional loop. The lateral sensitivity $\left(S_{\mathrm{L}}\right)$, which was related to the normal sensitivity $\left(S_{\mathrm{N}}\right)$, was calculated from $S_{\mathrm{L}}=\left(3 l_{\text {tip }} / 2 l\right)\left(d V_{\mathrm{N}} / d \mathrm{~V}_{\mathrm{L}}\right) S_{\mathrm{N}}$ based on probe's dimension as follow. ${ }^{1}$ Here, $l_{\text {tip }}, l, V_{\mathrm{N}}$ and $V_{\mathrm{L}}$, are tip height, cantilever length, normal and lateral deflection signals in voltage, respectively.

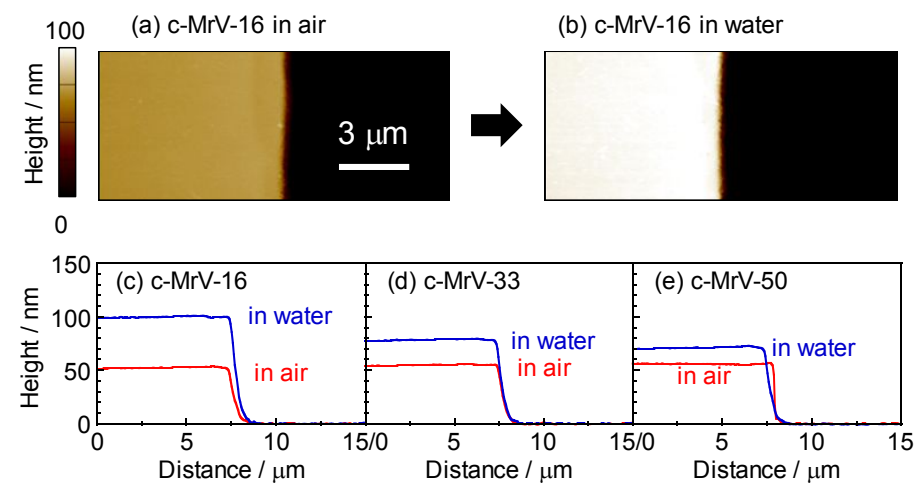

Figure S1. Topographic images for a c-MrV-16 film, in which a part was cut by a blade, observed in (a) air and (b) water, respectively. Height profiles for (c) c-MrV-16 (d) c-MrV-33 and (e) c-MrV50 films in air and water.

Table S1. Thickness and swelling ratio of c-MrV films obtained by AFM measurements. For comparison, the swelling ratio obtained by neutron reflectivity (NR) measurements are shown.

\begin{tabular}{|c|c|c|c|c|}
\hline \multirow{2}{*}{ samples } & \multicolumn{2}{|c|}{ film thickness / nm } & \multirow{2}{*}{$\begin{array}{c}\text { swelling ratio } \\
\text { by AFM }\end{array}$} & \multirow{2}{*}{$\begin{array}{c}\text { swelling ratio } \\
\text { by NR }\end{array}$} \\
\hline & in air & in water & & \\
\hline c-MrV-16 & $52.1 \pm 1.5$ & $99.7 \pm 3.3$ & $1.91 \pm 0.08$ & 1.96 \\
\hline c-MrV-33 & $54.9 \pm 1.6$ & $78.4 \pm 3.5$ & $1.43 \pm 0.08$ & 1.46 \\
\hline $\mathrm{c}-\mathrm{MrV}-50$ & $55.9 \pm 1.6$ & $70.0 \pm 2.0$ & $1.25 \pm 0.05$ & 1.33 \\
\hline
\end{tabular}


Panels (a) and (b) of Figure S1 show topographic images for a film of cross-linked poly(2methoxyethyl vinyl ether) containing 2-(vinyloxy)ethyl methacrylate (VEM) unit (c-MrV) with a VEM content of $16 \mathrm{~mol} \%$, in which a part was cut by a blade, observed in (a) air and (b) water. The zero height corresponds to the surface of the scratched area, or the substrate surface. Panels (c), (d) and (e) of Figure S1 illustrate height profiles for the c-MrV films in air and water. The film thickness was defined as an average step height excluding an edge rim part and summarized in Table S1.

\section{Analysis of neutron reflectivity}

Figure S2(a) shows NR curves for c-MrV films in a dried state. Open symbols are experimental data and solid lines denote calculated reflectivity based on the model scattering length density $(b / V)$ profiles shown in Figure S2(b). The $(b / V)$ value in the internal region increased with increasing VEM content, which was correlated with the cross-linking density in the film. The roughnesses of the c-MrV-16, -33 and -50 films were $1.3,1.6$ and $1.6 \mathrm{~nm}$, respectively. They were in good agreement with those obtained by AFM observations.
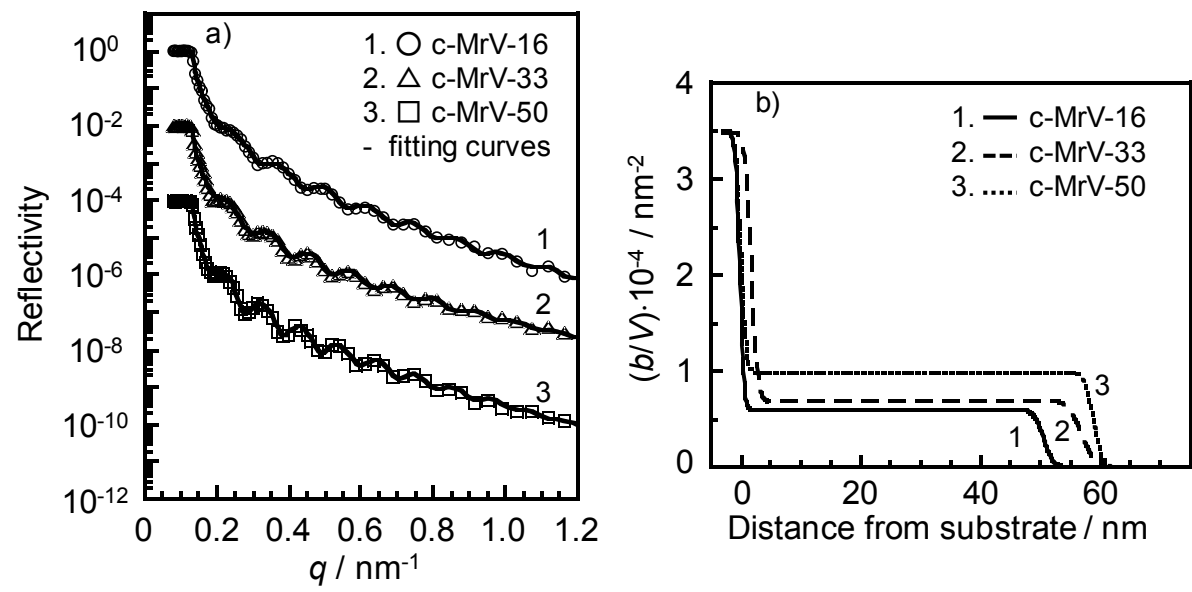

Figure S2. (a) Neutron reflectivity for c-MrV-16, -33 and -50 films in a dried state. Open symbols are experimental data and solid lines denote calculated reflectivity on the basis of (b) model scattering length density $(b / V)$ profiles.

Figure $\mathrm{S} 3$ shows NR curves for c-MrV film in $\mathrm{D}_{2} \mathrm{O}$ and the model scattering length density 
$(b / V)$ profiles where symbols denote experimental data sets. Solid lines in the panel (a) of Figure S3 denotes the best-fit calculated curves based on the model $(b / V)$ profiles using error function to describe the $\mathrm{D}_{2} \mathrm{O}$ interface as shown in the panel (b). Similarly, the solid lines in the panel (c) shows the best-fit calculated curves based on the model $(b / V)$ profiles using hyperbolic tangent to describe the $\mathrm{D}_{2} \mathrm{O}$ interface as shown in the panel (d). To determine the most appropriate model, $\chi^{2}$ values for the fitting were evaluated and listed in Table S2. The model using parabolic function provided the minimum value of $\chi^{2}$. The fitting results are shown in the main text.
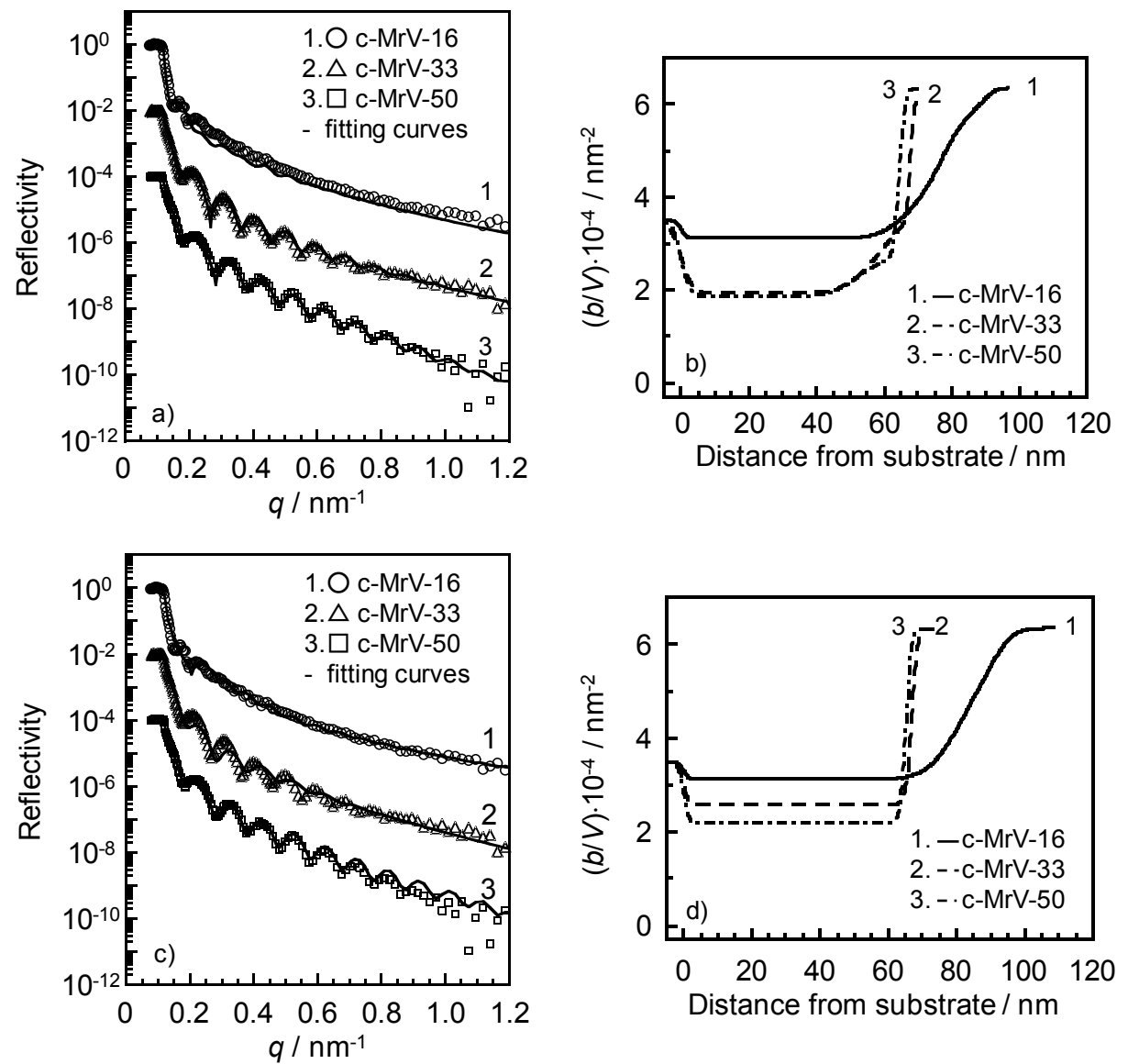

Figure S3. (a, c) Neutron reflectivity for c-MrV-16, -33 and -50 films in $\mathrm{D}_{2} \mathrm{O}$. Open symbols depict experimental data; solid lines are calculated reflectivity based on $(b / V)$ profiles. (b, d) Model scattering length density $(b / V)$ profiles using (b) error function and (d) hyperbolic function, respectively. 
Table S2. The $\chi^{2}$ values for the fitting analyses.

\begin{tabular}{cccc}
\hline Sample & error $/ 10^{-2}$ & $\begin{array}{c}\text { hyperbolic tangent } \\
/ 10^{-2}\end{array}$ & parabolic $/ 10^{-2}$ \\
\hline c-MrV-16 & 13 & 7.5 & 2.1 \\
c-MrV-33 & 8.1 & 6.2 & 0.91 \\
c-MrV-50 & 5.4 & 4.0 & 1.1 \\
\hline
\end{tabular}

\section{Reference}

1. Marti, O. Nanotribology - Friction on a Nanometer-Scale. Phys. Scr. 1993, T49B, 599-604. 